\title{
Sex Ratio Is Synergistically Modulated by Local Resource Competition and Enhancement: the Case of a Primitively Eusocial Wasp Under Female Philopatry
}

Koji Tsuchida ( $\nabla$ tsuchida@gifu-u.ac.jp )

Gifu University https://orcid.org/0000-0001-9359-5573

Norio Ishiguro

Gifu University Faculty of Applied Biological Science Graduate School of Agriculture: Gifu Daigaku Oyo Seibutsu Kagaku Gakubu Daigakuin Oyo Seibutsu Kagaku Kekyuka

Fuki Saito-Morooka

Ibaraki Daigaku Rigakubu

Jun-Ichi Kojima

Ibaraki Daigaku Rigakubu

Philip Spradbery

XCS Consulting

\section{Research}

Keywords: Sex ratio, enhancement, eusocial wasp, female philopatry

Posted Date: January 5th, 2021

DOI: https://doi.org/10.21203/rs.3.rs-138286/v1

License: (c) (i) This work is licensed under a Creative Commons Attribution 4.0 International License.

Read Full License 


\section{Abstract}

\section{Background}

In animals, the offspring sex ratio is modulated by kin conflict and cooperation, and determining the ratio is a main concern in evolutionary biology. Male competition for access to local mates is predictive of a female-biased sex ratio in the offspring (local mate competition; LMC). Conversely, female competition for access to local resources is predictive of a male-biased sex ratio in the offspring (local resource competition; LRC). However, several factors other than competition should synergistically operate in realworld populations. In the Australian paper wasp Ropalidia plebeiana, LRC and local resource enhancement (LRE) may operate simultaneously. To determine whether this is the case, we evaluated colony sex ratios and examined whether competition and/or enhancement operates at the population level in this species.

\section{Results}

In spring, many foundress queens started their colonies by comb-cutting, in which nest combs from the previous season were divided into several combs to be reused. Genetic relatedness among foundresses did not differ before and after comb-cutting. Relatedness among foundresses was 0.339 , whereas relatedness among new foundresses was 0.589 , revealing nearly functional monogyny. The global $F_{S T}$ value calculated with mtDNA markers was higher than that calculated with microsatellite markers, even after we corrected for differences in effective population sizes between sexes. This finding indicates female philopatry, which was also confirmed by mark-release-recapture before and after the hibernation of new foundresses. The colony sex ratio of reproductives became slightly biased toward males in larger colonies. In addition, both the number of foundresses and number of workers were positively associated with the number of reproductives, which indicates that LRE was also operating.

\section{Conclusions}

Our results suggest that although the population structure seems to meet the requirements of LRC, the sex ratio is not modulated solely by LRC. Instead, the availability of female helpers at the founding stage likely mitigates the sex ratio predicted by LRC through LRE. Thus, LRC at the founding stage and LRE at the reproductive stage synergistically modulate the colony sex ratio in $R$. plebeiana.

\section{Introduction}

Social hymenopterans have been the subject of both theoretical and empirical studies of sex ratio and kin selection [1]. Queen-worker conflict plays a pivotal role in determination of the sex ratio of reproductives (future queens and males). Workers favor a female-biased sex ratio ( 3 females: 1 male) in both monogynous and monandrous colonies because of the asymmetric relatedness introduced by haplodiploidy [2]. If colonies vary in terms of queen mating frequency, a split sex ratio is favored; colonies headed by a queen with a lower mating frequency should concentrate on only gyne (future queen) 
production, and those with queens that mate more often should focus on male production [3]. However, worker control of the sex ratio has not yet been confirmed in social insects with an annual life cycle, because the time required for worker control to evolve may not be available for such insects [4].

Conflict over access to local mates and resources also affects the sex ratio. When sons compete for access to local mates, a female-biased sex ratio (in the progeny) is favored by local mate competition (LMC) $[5,6]$. In contrast, when daughters compete for access to local resources, a male-biased sex ratio (in the progeny) is favored by local resource competition (LRC) $[7,8]$. These two types of competition are associated with different dispersal strategies; males disperse less than females (male philopatry) in the context of LMC, whereas females disperse less than males (female philopatry) in the context of LRC.

In addition to LMC and LRC, mutual helping among members of the same sex under local resource enhancement (LRE) can also affect the offspring sex ratio. LRE operates in facultatively eusocial insects, in particular, at the founding stage [9-11], where a female-biased sex ratio is favored when female helpers are more helpful in cooperatively breeding species [12]. Such enhancement is also evident in eusocial insects, particularly in polygynous societies, in which several founding queens cooperatively defend their brood across many eusocial insect groups [11, 13-21]. In general, within a species, polygyny is more advantageous than monogyny, especially during the founding stage before worker emergence, and colony productivity is positively associated with the founding group size [22-24].

The Australian paper wasp Ropalidia plebeiana provides a fascinating opportunity to evaluate LRC in the field. This species has an annual life cycle and founds a nest with multiple foundress queens [25]. The overwintered foundresses cooperatively start their colonies in an unusual fashionthey often reuse the nest combs season after season, and the combs from the previous season are usually cut into several smaller combs during the founding stage [26]. Furthermore, this species forms huge nesting aggregations (many hundreds or thousands of nests) that are re-used over many years; previously recorded aggregations [27] are still present decades later at the same location (Fig. 1). According to Itô and Higashi [28], these massive aggregations sometimes consist of several thousand combs hung side by side, and suggested that comb-cutting might reflect differences in relatedness. Such considerations and facts raise interesting ecological questions, as follows:

First, is comb-cutting based on relatedness (as suggested by Itô and Higashi [28])? If so (via nepotism), the relatedness among foundresses should increase after comb-cutting. Second, does LRC operate in the nesting aggregations? The fact that aggregations are relatively long-lasting leads us to hypothesize that foundresses compete over access to resources (nesting sites) and that sex ratio should be male-biased. Third, do LRC and LRE operate simultaneously? LRE with its polygynous advantage at the founding stage can balance with LRC through population structure at the reproductive-producing stage. To answer these questions, we conducted a field census and laboratory work to reveal the genetic structure of this species at the colony and population levels.

\section{Materials And Methods}




\section{Colony census}

Ropalidia plebeiana constructs massive nest aggregations (Fig. 1). We conducted a colony census of 26 colonies in a population at Dinner Creek in New South Wales, Australia (location data in Table S1). These routinely censused colonies (RCCs) were censused approximately every 3 days from mid-October 2001 to the end of March 2002; newly emerged adult females in the RCCs were individually marked with paint. During the paper wasp life cycle, foundresses begin nest construction and produce workers, gynes, and males. A foundress is assigned a queen after workers begin to emerge if she is the egg-producer at that time. Gynes that are candidate female foundresses in the following season were designated as females that emerged after February 21, 2002, for the following reason: we found 59 marked and overwintered foundresses again on September 16,2002, and the mean date of emergence of the first gyne marked for 19 colonies was February 21, 2002. Newly emerged males were collected with forceps and immediately stored in $99 \% \mathrm{EtOH}$. The mean date of emergence of the first male in 24 colonies was March 2, 2002. The original queen(s) of the RCCs were collected in the same manner in March, if still alive, and stored in $99 \%$ $\mathrm{EtOH}$. Workers that emerged before February 21 were collected during March. At the end of March, we collected the whole RCC and counted the number of pupae. Then, we collected all pupae of each colony and stored them in $99 \% \mathrm{EtOH}$. We checked and sexed the collected insects according to morphological differences in antennae. We estimated the sex ratio of reproductives (males/[males + new gynes]) for each colony from these data. We genotyped some of these female pupae as new gynes.

In addition, we individually marked 960 foundresses in 111 arbitrarily censused colonies with paint before worker emergence at Dinner Creek. From October 2001 to February 2002, we arbitrarily collected the colonies, including all colony members of foundresses with marks and emerged workers without marks, and stored them in $99 \% \mathrm{EtOH}$. After each round of collection, we visually checked whether each colony had been divided into subcolonies by comb-cutting, because it was possible to observe comb-cutting during each colony census. The number of colonies was 16 before comb-cutting and 36 after combcutting.

All collected female adults were dissected under a microscope, and their ovarian condition was noted as follows: 0 , undeveloped; 1 , slightly developed; 2 , one mature egg; 3 , more than two mature eggs.

\section{Sample collection for population structure}

We collected $14-40$ adults from each of the 14 populations (total: 309 ) in 2008 , including the population at Dinner Creek, where we conducted the colony census using insect nets and stored them in $99 \% \mathrm{EtOH}$.

\section{Genetic analyses}

Collected individuals were transported back to the laboratory and stored in $99 \% \mathrm{EtOH}$ at $-20{ }^{\circ} \mathrm{C}$ until DNA extraction. The excised legs of adults and pupae were frozen in liquid nitrogen and smashed with a pestle in 1.5-mL tubes, then mixed with $50 \mu \mathrm{L}$ extraction buffer containing $150 \mathrm{mM} \mathrm{NaCl}, 10 \mathrm{mM}$ Tris- $\mathrm{HCl}(\mathrm{pH}$ 8.0), $1.0 \mathrm{mM}$ EDTA, $10 \mu \mathrm{g}$ proteinase $\mathrm{K}$, and $40 \mu \mathrm{g}$ Chelex 100 (Bio-Rad Laboratories). The tubes were 
incubated at $56^{\circ} \mathrm{C}$ for $2 \mathrm{~h}$ and at $99.9^{\circ} \mathrm{C}$ for $3 \mathrm{~min}$ [29]. Then each solution was precipitated with ethanol and maintained in TE buffer at $4{ }^{\circ} \mathrm{C}$.

\section{Genotyping for $\mathrm{COI}$}

Polymerase chain reaction (PCR) was performed with $1 \mu \mathrm{L}$ diluted genomic DNA (approximately $1 \mathrm{ng}$ ) in a mixture consisting of $1 \mu \mathrm{L}$ primer mix $(2.5 \mu \mathrm{M}), 0.1 \mu \mathrm{L} 10 \mathrm{mM}$ dNTP mix, $0.05 \mu \mathrm{L}$ Taq polymerase (5 units/ $\mu \mathrm{L}$, Ex Taq; TaKaRa), $1 \mu \mathrm{L} 10 \times$ buffer (provided with the polymerase, containing $1.5 \mathrm{mM} \mathrm{MgCl}_{2}$ ), and $6.85 \mu \mathrm{L}$ distilled water with a total volume of $10 \mu \mathrm{L}$. PCR was performed using a thermal cycler (Gene Amp PCR System 2700; Applied Biosystems). After denaturation for 4 min at $94^{\circ} \mathrm{C}$, the samples were subjected to $30-35$ cycles of $1 \mathrm{~min}$ at $94^{\circ} \mathrm{C}, 1 \mathrm{~min}$ at the annealing temperatures appropriate for the particular primer pair, and $45 \mathrm{~s}$ at $72^{\circ} \mathrm{C}$, with a final extension for $7 \mathrm{~min}$ at $72{ }^{\circ} \mathrm{C}$. We used five primer pairs (Table S2). The products were electrophoresed in 8\% polyacrylamide gel and visualized by silver staining [30]. Genotype scoring and data entry were performed twice, and the scores were compared. Discrepancies were checked, and if necessary, the sample was reanalyzed.

The cytochrome oxidase subunit I (COI) gene was amplified and sequenced for the population samples. PCR fragments were produced with the mtDNA primers C01-LCO and C01-HCOoutout [31]. At the first step, double-stranded PCR products were generated with a PCR mixture containing $2 \mu \mathrm{L}$ genomic DNA, $5 \mu \mathrm{L}$ primer mix (0.8 pmol/ $\mu \mathrm{L}), 2.5 \mu \mathrm{L}$ 10' PCR buffer, $2 \mu \mathrm{L} 2.5 \mathrm{M}$ dNTP, $10.375 \mu \mathrm{L}$ distilled water, and $0.125 \mu \mathrm{L}$ Taq DNA polymerase (5 units/ $\mu \mathrm{L}$, Ex Taq; TaKaRa) with a total volume of $22 \mu \mathrm{L}$. The PCR program was: 5 min of denaturing at $94{ }^{\circ} \mathrm{C}$ followed by 40 cycles of $15 \mathrm{~s}$ at $94{ }^{\circ} \mathrm{C}, 5 \mathrm{~s}$ at $46^{\circ} \mathrm{C}$, and $30 \mathrm{~s}$ at $68^{\circ} \mathrm{C}$. The reaction was terminated with 7 min of elongation at $72^{\circ} \mathrm{C}$. An aliquot of the PCR products from this first step was used as the template for a second PCR with only one of the two primers added to produce a single-stranded PCR product. The second PCR mixture included $4 \mu \mathrm{L}$ DNA template (PCR product from the first step), $2 \mu \mathrm{L}$ primer (0.8 pmol/ $\mu \mathrm{L}), 3.5 \mu \mathrm{L}$ sequencing buffer, and $0.5 \mu \mathrm{L}$ BigDye Terminator Ready Reaction Mix (Applied Biosystems) in a total volume of $10 \mu \mathrm{L}$. The single-stranded PCR products were sequenced with dye-labeled terminators with the ABI Prism BigDye Terminator Cycle Sequencing Ready Reaction Kit (Applied Biosystems) and 3100 DNA analyzer (Perkin-Elmer). PCR fragments were sequenced in both directions to ensure their accuracy.

\section{Analyses of Population structure}

For microsatellite analyses, we developed specific primers for $R$. plebeiana. We used four specific primer pairs and one new pair specific for $R$. revolutionalis (Rrev 188; [32]) to analyze the population and colony structures of $R$. plebeiana. For the microsatellite data set, we checked the existence of null alleles across the loci with MICRO-CHECKER [33] using genotyping data from 309 adults from the 14 populations. Departures from Hardy-Weinberg assumptions were calculated using GenAlEx ver. 6 [34]. Linkage disequilibrium was tested with Genepop ver. 1.2 [35]. We sampled the 14 populations using arbitrary netting by hand. Therefore, we may have captured some kin groups. To exclude this possibility, we detected full-sib relationships using Kinship [36] and removed them from our data. We ran the software 
assuming "full-sib" as the primary hypothesis and "unrelated" as the null hypothesis, with a Type I error of less than 0.05 .

For both the microsatellite and mtDNA data sets, we quantified genetic variation between hierarchical levels of populations (among regions, among populations within regions, and within populations) and their fixation indices using analysis of molecular variance [37] in GenAlEx ver. 6 [34]. To examine mtDNA haplotype differentiation, this method yields analogs of $\mathrm{F}$ statistics, called $\Phi$ statistics, as defined by Michalakis and Excoffier [38]. We determined the probabilities that the molecular variance and fixation indices at different levels were significant and positive (indicating differentiation) using permutation analyses of 1,000 randomly permuted data sets. $F_{S T}$ (for microsatellites) and $\Phi_{S T}$ (for mtDNA) show the overall genetic variation among populations, indicating a sex-biased dispersal pattern. We evaluated genetic differentiation among populations with pairwise $F_{S T}$ with 10,000 permutations using GenAlEx ver.

6. Pairwise $F_{S T}$ was used to investigate the impact of interpopulation geographic distance on genetic differentiation using an isolation-by-distance (IBD) model [39]. Significant correlations between genetic differentiation ( $\mathrm{F}_{\mathrm{ST}}$ values for both markers) and the corresponding geographic distance were assessed using a Mantel test with 10,000 permutations. For geographic distances, straight-line distances (in kilometers) between paired members of the 14 populations were considered.

We applied Bayesian clustering in STRUCTURE ver. 2.0 [40] as an alternative measure of the degree of genetic differentiation using the microsatellite data set to delineate the number of genetically identified clusters $(K)$ and assign individuals to clusters without using prior information about their locations of origin. We used an admixture model with correlated allele frequencies [41] for $\mathrm{K}$ values 1 (panmixia) to 10, performing 10 runs for each $\mathrm{K}$ value with 1,000,000 Markov chain Monte Carlo iterations and a burn-in period of 100,000 iterations. We estimated the likelihood of $K$ for $K=1-10$; the highest likelihood indicated the most probable number of populations. We calculated an ad hoc criterion of $K$ [42] to determine the optimal K.

\section{Analyses of Colony structure}

We genotyped four classes of females, namely, foundresses, nestmate workers, new gynes, and foundresses of the following season. To genotype foundresses of the following season, we collected females on September 15 and 16 in 2002. For new gynes, we genotyped the pupae as well as adults of each colony. We calculated coefficients for genetic relatedness and inbreeding using Relatedness ver. 4.2c [43]. Standard errors were based on jackknifing over colonies [44]. Colonies were weighted equally in all analyses.

If not otherwise specified, all statistical analyses were performed in R ver. 3.4.1 [45].

\section{Results}

\section{Population structure}


Using Kinship [36], we found that 161 of the 309 foundresses collected via arbitrary hand-netting from the 14 populations were in full-sib relationships (relatedness $=0.75$ ) under haplodiploidy. Therefore, we removed these data from further analyses of population structure.

No significant departures from the Hardy-Weinberg equilibrium were detected in the five loci of the remaining 148 individuals after sequential Bonferroni correction. Similarly, no significant linkage disequilibrium or null alleles were detected for the five loci in the 14 populations. These results show that the five loci were useful markers for studying population structure in this species.

We did not detect any genetic structure in the 14 populations using STRUCTURE (Fig. S1). In the microsatellite dataset, the global $\mathrm{F}_{\mathrm{ST}}$ was $0.007(P=0.152), \mathrm{F}_{\mathrm{IS}}$ was $0.045(P=0.033)$, and $\mathrm{F}_{\mathrm{IT}}$ was 0.052 $(P=0.017)$, which suggest no apparent subdivisions in the populations but a slight trend toward inbreeding within each population.

We found 20 haplotypes of COI (accession nos. LC533797-LC533806, and LC554438-LC554447). In the COI haplotype dataset, the global $\Phi_{\mathrm{ST}}$ was $0.102(P=0.001)$, indicating apparent subdivisions in the populations. Without a sex-biased gene flow, the following relationship between microsatellites (represented by $\left.\mathrm{F}_{\mathrm{ST}}\right)$ and $\mathrm{COI}\left(\Phi_{\mathrm{ST}}\right)$ is expected [46]:

$$
\Phi_{S T}=\frac{4 F_{S T}}{\left(1+3 F_{S T}\right)}
$$

The expected $\Phi_{S T}(0.027)$ was lower than the observed $\Phi_{S T}(0.102)$, which implies that female philopatry is the rule in this species.

IBD relationships based on the microsatellite dataset and $\mathrm{COI}$ are shown in Fig. 2. IBD relationships were present based on the microsatellite dataset (Mantel test, $r_{s}=0.497, P=0.01$ ) but not on the COI dataset $\left(r_{s}=-0.014, P=0.51\right)$, which indicates that gene flow and genetic drift in each population were balanced in terms of microsatellites. By contrast, genetic drift was more prominent than gene flow for mtDNA, which indicates that in each subpopulation, the population structure of females was more subdivided by genetic drift than that of males. This result did not contradict the result mentioned above regarding the $\mathrm{F}_{\mathrm{ST}}$ and $\Phi_{\mathrm{ST}}$ values of the microsatellite and mtDNA datasets.

We found 59 females marked in the previous season again in the following spring of September 2002, when comb-cutting had not yet started. We counted the number of combs between the natal comb and the comb on which each foundress was found the next spring (Fig. 3 , inset). The relationship between the number of foundresses found the next spring, and the number of combs from the natal comb is shown in Fig. 3. Most foundresses were on the natal comb or the next comb over, suggesting female philopatry. The frequency distribution did not differ from a negative binomial distribution $\left(\chi^{2}\right.$ cal $=3.458, \mathrm{df}=1, P=$ $0.063)$; thus, the distribution is indicative of contagious distribution.

\section{Colony structure}


We compared the genetic relatedness of foundresses before and after comb-cutting (Fig. 4). It was 0.213 $\pm 0.047(n=16)$ before comb-cutting and $0.299 \pm 0.049(n=36)$ afterward. There was no significant difference in relatedness (Welch's t-test, $P=0.197$ ). Figure 5 shows the relatedness among foundresses before worker emergence, among workers, among new gynes, and among new foundresses the next spring. Relatedness was significantly higher among new gynes $(0.589 \pm 0.053)$ than among foundresses ( $0.339 \pm 0.047$, Welch's t-test and sequential Bonferroni correction, $P=0.0038)$. However, relatedness among foundresses did not differ significantly from relatedness among workers $(0.235 \pm 0.030$, Welch's ttest, $P=0.033)$ or among foundresses the next spring $(0.240 \pm 0.054$, Welch's t-test, $P=0.098)$ after sequential Bonferroni correction. The frequency distribution of pairwise relatedness among foundresses (Fig. 6) exhibited a broad range of relatedness values and many pairs with zero relatedness. Here, we hypothesized that the pairs comprised only full-sibs and pairs with zero relatedness, and thus we can estimate that unrelated pairs made up $54.8 \%$ of the pairs of foundresses.

We used GLM to analyze the effects of the number of workers and relatedness among workers on the colony sex ratio with a binomial distribution (Table S3). The number of workers had a positive effect on the sex ratiothe more workers there were in a colony, the more likely the sex ratio was to be biased toward males (Fig. 7). The mean colony sex ratio was slightly biased toward females $(0.44 \pm 0.03, n=24)$, and the population sex ratio was 0.50 . Because the dry weight of new gynes was heavier than that of males (new gynes: $10.51 \pm 0.26 \mathrm{mg}, \mathrm{n}=20$; males: $9.71 \pm 0.29 \mathrm{mg}, \mathrm{n}=20$ ), the population investment ratio was 0.48 .

We also used GLM to analyze the effects of the number of foundresses in spring and that of workers on the total productivity of reproductives (new gynes + males) with a Poisson distribution (Table S4). Both numbers had positive effects on total productivity, which indicates that colonies with more foundresses were more productive.

The relationships between the number of workers and both new gynes and males produced by each colony are shown in Fig. 8. As predicted by LRC, the slope of the regression line was greater for males than for gynes $\left(\mathrm{t}_{\mathrm{cal}}=3.22, P=0.002\right)$.

\section{Discussion}

\section{Population structure and sex ratio}

The paper wasp R. plebeiana constructs dense aggregations of nests year after year at patchy locations [25-27]. The combs within such aggregations are generally reused in the next season [26]. This life history strongly implies that suitable locations for nesting are likely restricted within these patchy locations along the coast of New South Wales, where temperate rainforests prevail. Such restrictions likely force foundresses to compete for access to nesting sites, a condition that can lead to LRC. 
We used molecular markers to study the population and colony structure of the primitively eusocial wasp R. plebeiana. Although we did not detect IBD relationships based on mtDNA, we found these relationships using microsatellites (Fig. 2), suggesting that gene flow and genetic drift in each population were not balanced in terms of maternally inherited mtDNA but were balanced in terms of the biparental nuclear DNA microsatellites. The estimated global $\Phi_{S T}$ for mtDNA was higher than $F_{S T}$ for microsatellites, even when we corrected the value with the difference in the effective population size between the two markers. These results suggest that new foundresses disperse less than males upon mating and that females have a more structured population than males. This result is in line with our mark-release-recapture study from the end of the colony season to the start of colony founding; foundresses started their founding activity at either their natal combs or combs nearby in a contagious fashion (Fig. 2). All of these results strongly imply female philopatry in this species. Female philopatry accords with the higher $\Phi_{S T}$ for mtDNA than $\mathrm{F}_{\mathrm{ST}}$ for microsatellites and agrees with the conditions that are prerequisites for LRC.

The colony sex ratio in reproductives became significantly biased toward males in larger colonies (Fig. 7). The constant female hypothesis in LRC predicts a colony sex ratio increasingly biased toward males when competition over access to resources among females prevails [7, 8], which suggests that LRC likely operates. However, although this relationship was significant, it was moderate; we did not observe sex ratios that were extremely biased toward males in larger colonies as LRC predicted. This evidence suggests that certain factors counteract LRC in these populations.

One factor that might mitigate LRC is LRE, which comprises positive interactions among cooperative breeding females [9-11]. We found such a cooperative breeding advantage in $R$. plebeianathe number of foundresses in spring had a positive effect on total productivity (Table S4); i.e., colonies with more foundresses were more productive. Cooperative nest founding (polygyny) is more advantageous than single founding (monogyny), as polygyny results in greater productivity and more effective defense against parasites compared to monogyny $[15,20,47-50]$. LRE counteracts LRC in $R$. plebeiana, and therefore we did not observe sex ratios that were extremely biased toward males in the larger colonies.

Another factor that may mitigate LRC is caste plasticity [51, 52], such that protogyny is associated with a sex ratio moderately biased toward females. The caste plasticity hypothesis proposes that females are produced before males (protogynous production) whenever there is uncertainty regarding the optimal time to begin producing reproductives. Thus, we might expect that the sex ratio would be biased toward females because females may become either workers or gynes (future queens) until late in their development and respond more flexibly than males [51]. In this study, the average date of emergence of reproductive females in $R$. plebeiana was February 21, 2002, and that of males was March 2, 2002. Such protogynous production might have also counteracted the male-biased sex ratio favored by LRC.

In a relatedness framework, worker control of investment predicts a female-biased sex ratio in monogynous and monandrous colonies. Still, such sex ratios have not always been reported, especially in primitively eusocial wasps of the annual life cycle $[4,53]$. The lack of a female-biased sex ratio may be because worker control of investment is characteristic of advanced eusociality (e.g., swarm founding). In 
the primitively eusocial paper wasp Polistes chinensis antennalis, queen control of investment is observed, even when workers lay haploid eggs in queen-right conditions [53]. In R. plebeiana, many workers retain developed ovaries, suggesting that they likely contribute to male production in each colony. This finding may provide an opportunity for queens to make the sex ratios more biased toward females to compensate for male production by workers (i.e., sex ratio compensation) [54]. Further studies are needed to determine whether worker reproduction contributes to adult male production in this species.

\section{Colony genetic structure}

Makino et al. [26] reported that $R$. plebeiana reuses old combs from the previous season and that many combs are gnawed into several independent combs by females. Following this observation, they hypothesized that each comb is nepotistically divided into smaller combs based on differences in relatedness: Groups of foundresses are divided into smaller groups according to a closer kin relationship. Although we tested this hypothesis in this study using genetic markers, we failed to detect such higher kin group formation at the founding stage, which shows that nepotistic behavior is lacking at this stage in $R$. plebeiana. Although the inclusive fitness theory favors nepotistic behavior, it is observed less frequently than expected in eusocial insects [55] (but see Hannonen and Sundström [56]). Nepotistic behavior is rarely observed in eusocial insects, likely because the fine-scale recognition cue is costly to maintain. Parsons et al. [57] used genetic markers to analyze large-scale population structure in Polistes dominula. They found that a significant fraction of wasps chose nests that did not contain sisters, despite sisters being present in a nearby nest. We observed a slight upward shift in relatedness between before and after comb-cutting $(0.299-0.213=0.086)$; such relatedness is quite subtle. Such a small benefit (indirect fitness) does not sufficiently explain subordinate female helping behavior within subdivided combs. Although further studies are needed, present evidence suggests that subordinate foundresses are unlikely to benefit sufficiently from indirect fitness through relatedness to dominant ones by comb-cutting.

The genetic relatedness among foundresses and workers was 0.235 ; the effective number of mothers for workers was 3.94 according to the equation $\left[R_{d}=3 / 4 N+R_{q} / 4(1-1 / N)\right]$, where $R_{d}$ = relatedness among daughters, $R_{q}=$ relatedness among foundresses, and $N=$ effective number of mothers [58]. However, the genetic relatedness among new gynes was 0.589 , and the number of mothers for new gynes was 1.30 . This means that colonies of $R$. plebeiana are functionally monogynous and that the majority of subordinate foundresses do not contribute offspring production to the next generations. The frequency distribution of pairwise relatedness among foundresses showed that many foundress pairs exhibited zero relatedness (Fig. 6). Such non-kin helpers can achieve sufficient fitness if they can inherit colonies, as in $P$. dominula $[59,60]$. However, such an opportunity might be quite limited in $R$. plebeiana because we did not observe any queen turnover in our RCCs (data not shown). Another possibility is that the fate of the foundress is likely determined in a pre-imaginal fashion. In $R$. marginata, the future queen is determined before the existing queen is lost. Her nestmates recognize her as the future queen; the queen's pheromonal control over the colony is more important than physical aggression [61, 62]. In R. plebeiana, the future queens behave docilely at the pre-worker stage and never forage (Tsuchida, unpublished data), 
which implies that the future queens may also signal her presence via pheromones. Such a possibility should be examined in future chemical studies.

In this study, we detected female philopatry, a prerequisite for LRC, in both mark-release-recapture and gene flow studies. Female philopatry was also observed in another study of $R$. plebeiana [63]. Besides, we found that the colony sex ratio becomes more biased toward males in larger colonies, suggesting that LRC operates in R. plebeiana. However, we did not observe an extremely male-biased sex ratio, which suggests that selective forces counteract LRC. One of these forces is LRE, in which cooperative female helpers may enhance colony performance at the founding stage. LRC leading to a male-biased sex ratio may counteract LRE leading to a female-biased sex ratio, and as a result, the population sex ratio evens out. Although genetic relatedness among foundresses increased slightly at the colony level, these values did not differ significantly before and after comb-cutting. Such a slight increase seems insufficient for explaining indirect fitness gain in subordinate foundresses. In general, no worker control of the sex ratio has been confirmed in the primitively eusocial paper wasps. The relatively short annual colony cycle in such wasps may constrain worker control of the sex ratio; colony maintenance is a more important driving force than reproduction, mainly because, unlike in perennial species, they have only one chance at it [64]. Such seasonal constraints may not allow for the evolution of the adaptive traits expected in theory.

\section{Conclusions}

The restricted nesting sites and strong female philopatry observed in this study indicate that LRC likely operate in R. plebeiana. We found strong female philopatry using both mark-release-recapture during the founding stage and molecular markers. The colony sex ratio increased significantly with colony size, which indicates that LRC operates. However, we did not observe an extremely male-biased sex ratio, which means that LRE likely operates as well. This result suggests that LRC leading to a male-biased sex ratio is counteracted by LRE leading to a female-biased sex ratio. Many combs used in the previous season are divided into smaller combs by comb-cutting at the founding stage. However, this behavior does not seem to be based on relatedness, and the subordinate indirect fitness benefit seems to be small. One reason why we did not find an adaptive reaction such as LRC and comb-cutting based on relatedness may have to do with the seasonal constraints of the annual life cycle, which do not allow sufficient time for $R$. plebeiana to evolve the adaptive reactions expected under LRC and kin selection.

\section{Declarations}

\section{DATA AVAILABILITY}

Requests to access the datasets should be directed to tsuchida@gifu-u.ac.jp

\section{ETHICS STATEMENT}

All material was collected in accordance with the ethical standards and laws of the institution and country in which the studies were conducted. 


\section{COMPETING INTERESTS}

The authors declare that they have no competing interests.

\section{FUNDING}

The fieldwork was supported by the Bilateral Researcher Exchange Program between the Japan Society for the Promotion of Science and the Australian Academy of Science (grant to KT). The laboratory work was partially supported by a grant from the Japan Society for the Promotion of Science (KAKENHI) no. $19 \mathrm{~K} 06836$ to KT.

\section{AUTHORS CONTRIBUTIONS}

KT conceived the study, and designed and performed the fieldwork. NI conducted DNA analyses. FS and JK collected samples and extracted DNA. PS supported the field work by KT, FS, and JK and discussed the results with KT. KT performed the DNA analyses and all statistical tests. KT wrote the paper, and all authors (except PS) read and approved the final manuscript.

\section{ACKNOWLEDGMENTS}

We express our sincere thanks to Koji Yajima and Morito Hino for their help with the laboratory work. We thank Nicole Fisher and John LaSalle for facilitating KT's stay in Canberra and his fieldwork. We obtained permission to transfer our samples from the Australian National Insect Collection, CSIRO, Canberra. Michael Schwarz kindly allowed KT to conduct laboratory work in Adelaide. We thank Adam Cronin for his comments on an earlier version of the manuscript. Sadly, PS passed away in 2019.

\section{References}

1. Mehdiabadi NJ, Reeve HK, Mueller UG. Queens versus workers: Sex-ratio conflict in eusocial Hymenoptera. Trends Ecol Evol. 2003;18:88-93.

2. Trivers RL, Hare H. Haplodiploidy and the evolution of the social insects. Science 1976;191:249-263.

3. Boomsma JJ, Grafen A. Interspecific variation in ant sex ratios and the Trivers-Hare hypothesis. Evolution 1990;44:1026-1034.

4. Tsuchida K, Suzuki T. Conflict over sex ratio and male production in paper wasps. Annal Zool Fenn. 2006;43:468-480.

5. Frank SA. Hierarchical selection theory and sex ratios. II. On applying the theory, and a test with fig wasps. Evolution 1985;39:949-964.

6. Yamaguchi Y. Sex ratios of an aphid subject to local mate competition with variable maternal condition. Nature 1985;318:460-462.

7. Clark AB. Sex ratio and local resource competition in a prosimian primate. Science 1978;201:163165. 
8. Frank SA. Variable sex ratio among colonies of ants. Behav Ecol Sociobiol. 1987;20:195-201.

9. Schwarz MP. Local resource enhancement and sex ratios in a primitively social bee. Nature 1988;331:346-348.

10. Cronin AL, Schwarz MP. Sex ratios, local fitness enhancement and eusociality in the allodapine bee Exoneura richardsoni. Evol Ecol. 1997;11:567-577.

11. Schwarz MP, Bull NJ, Hogendoorn K. Evolution of sociality in the allodapine bees: a review of sex allocation, ecology and evolution. Ins Soc. 1998;45:349-368.

12. Silk JB, Brown GR. Local resource competition and local resource enhancement shape primate birth sex ratios. Proc Roy Soc Lond B. 2008;275:1761-1765.

13. Lin N, Michener CD. Evolution of sociality in insects. Quart Rev Biol. 1972;47:131-159.

14. Evans HE. Extrinsic versus intrinsic factors in the evolution of insect sociality. BioScience 1977;27:613-617.

15. Gamboa GJ. Intraspecific defense: advantage of social cooperation among paper wasp foundresses. Science 1978;199:1463-1465.

16. Strassmann JE, Queller DC, Hughes CR: Predation and the evolution of sociality in the paper wasp Polistes bellicosus. Ecology 1988;69:1497-1505.

17. Gadagkar R. Demographic predisposition to the evolution of eusociality: A hierarchy of models. Proc Nat Acad Sci USA. 1991;88:10993-10997.

18. Hughes CR, Queller DC, Strassmann JE, Davies SK. Relatedness and altruism in Polistes wasps. Behav Ecol. 1993;4:128-137.

19. Makino S, Sayama K. Comparison of intraspecific nest usurpation between two haplometrotic paper wasp species (Hymenoptera: Vespidae: Polistes). J Ethol. 1991;9:121-128.

20. Gamboa GJ, Wacker TL, Duffy KG, Dobson SW, Fishwild TG. Defence against intraspecific usurpation by paper wasp cofoundresses (Polistes fuscatus, Hymenoptera: Vespidae). Can J Zool. 1992;70:2369-2372.

21. Itô Y. Behaviour and social evolution of wasps. The communal aggregation hypothesis. Oxford: Oxford University Press; 1993.

22. Cant MA, Field J. Helping effort and future fitness in cooperative animal societies. Proc Roy Soc Lond B. 2001;268:1959-1964.

23. Shreeves G, Cant MA, Bolton A, Field J. Insurance-based advantage for subordinate co-foundresses in a temperate paper wasp. Proc Roy Soc Lond B. 2003;270:1617-1622.

24. Liebert AE, Starks PT: Taming of the skew: transactional models fail to predict reproductive partitioning in the paper wasp Polistes dominulus. Anim Behav. 2006;71:913-923.

25. Itô Y, Yamane S, Spradbery JP. Population consequences of huge nesting aggregations of Ropalidia plebeiana (Hymenoptera: Vespidae). Res Popul Ecol. 1988;30:279-295.

26. Makino S, Yamane S, Itô Y, Spradbery JP. Process of comb division of reused nests in the Australian paper wasp Ropalidia plebeiana (Hymenoptera, Vespidae). Ins Soc. 1994;41:411-422. 
27. Richards OW. The Australian social wasps (Hymenoptera: Vespidae). Aust J Zool. 1978;Suppl 26:1132.

28. Itô Y, Higashi S. Spring behaviour of Ropalidia plebeiana (Hymenoptera: Vespidae) within a huge aggregation of nests. Appl Ent Zool. 1987;22:519-527.

29. Walsh PS, Metzger DA, Higuchi R. Chelex 100 as a medium for simple extraction of DNA for PCRbased typing from forensic material. BioTechniques 1991;10:506-513.

30. Bassam BJ, Caetano-Anolles G, Gresshoff PM. Fast and sensitive silver staining of DNA in polyacrylamide gels. Analy Biochem. 1991;196:80-83.

31. Pickett KM, Tolman GL, Wheeler WC, Wenzel JW. Parsimony overcomes statistical inconsistency with the addition of more data from the same gene. Cladistics 2005;21:438-445.

32. Henshaw MT, Crozier YC, Crozier RH. New microsatellite loci for the socially diverse paper wasp genus Ropalidia. Mol Ecol Notes 2003;3:641-643.

33. van Oosterhout C, Hutchinson WF, Wills DPM, Shipley P. MICRO-CHECHER: software for identifying and correcting genotyping errors in microsatellite data. Mol Ecol Notes 2004;4:535-538.

34. Peakall R, Smouse PE. GENALEX 6: Genetic analysis in Excel. Population genetic software for teaching and research. Mol Ecol Notes 2006;6:288-295.

35. Raymond M, Rousset F. GENEPOP (Version 1.2): Population genetics software for exact tests and ecumenicism. J Hered. 1995;86:248-249.

36. Goodnight KF, Queller DC. Computer software for performing likelihood tests of pedigree relationship using genetic markers. Mol Ecol. 1999;8:1231-1234.

37. Excoffier L, Laval G, Schneider S. Arlequin (version 3.0): An integrated software package for population genetics data analysis. Evol Bioinform. 2005;1:47-50.

38. Michalakis Y, Excoffier L. A generic estimation of population subdivision using distances between alleles with special reference for microsatellite loci. Genetics 1996;142:1061-1064.

39. Wright S. Isolation by distance. Genetics 1943;28:114-138.

40. Pritchard JK, Stephens M, Donnelly P. Inference of population structure using multilocus genotype data. Genetics 2000;155:945-959.

41. Falush D, Wirth T, Linz B, Pritchard JK, Stephens M, Kidd M, Blaser MJ, Graham DY, Vacher S, PerezPerez GI, et al. Traces of human migrations in Helicobacter pylori populations. Science 2003;299:1582-1585.

42. Evanno G, Regnaut S, Goudet J. Detecting the number of clusters of individuals using the software STRUCTURE: A simulation study. Mol Ecol. 2005;14:2611-2620.

43. Goodnight, K.F., 1996. Relatedness 4.2c Release, Rice University, Texas, U.S.A., http://gsoft.smu.edu/Gsoft.html.

44. Queller DC, Goodnight KF. Estimating relatedness using genetic markers. Evolution 1989;43:258275. 
45. R Development Core Team: R: A language and environment for statistical computing. Vienna, Austria: R Foundation for Statistical Computing; 2020. http://www.R-project.org.

46. Crochet P-A. Genetic structure of avian populations - allozymes revisited. Mol Ecol. 2000;9:14631469.

47. Klahn JE. Intraspecific comb usurpation in the social wasp Polistes fuscatus. Behav Ecol Sociobiol. 1988;23:1-8.

48. Katada S, Iwahashi O. Characteristics of usurped colonies in the subtropical paper wasp, Ropalidia fasciata (Hymenoptera: Vespidae). Ins Soc. 1996;43:247-253.

49. Yamane S: Ecological factors influencing the colony cycle of Polistes wasps. In: Turillazzi S, WestEberhard MJ, editors. Natural history and evolution of paper-wasps. Oxford: Oxford University Press; 1996.p. 75-97.

50. Clouse R. Some effects of group size on the output of beginning nests of Mischocyttarus mexicanus (Hymenoptera: Vespidae). Flor Entomol. 2001;84:418-425.

51. Strassmann JE. Female-biased sex ratios in social insects lacking morphological castes. Evolution 1984;38:256-266.

52. Suzuki T. Production schedule of males and reproductive females, investment sex ratios, and workerqueen conflict in paper wasps. Am Nat. 1986;128:366-378.

53. Tsuchida K, Saigo T, Nagata N, Tsujita S, Takeuchi K, Miyano S. Queen-worker conflict over male production and sex allocation in a primitively eusocial wasp. Evolution 2003;57:2365-2373.

54. Taylor PD. Sex ratio compensation in ant population. Evolution 1981;35:1250-1251.

55. Boomsma JJ, d'Ettorre P. Nice to kin and nasty to non-kin: revisiting Hamilton's early insights on eusociality. Biol Lett. 2013;9.

56. Hannonen M, Sundström L. Worker nepotism among polygynous ants. Nature 2003; 421:910.

57. Parsons PJ, Grinsted L, Field J. Partner choice correlates with fine scale kin structuring in the paper wasp Polistes dominula. PLoS one 2019;14:e0221701.

58. Strassmann JE, Queller DC, Solis CR, Hughes CR: Relatedness and queen number in the Neotropical wasp, Parachartergus colobopterus. Anim Behav 1991;42:461-470.

59. Leadbeater E, Carruthers JM, Green JP, Rosser NS, Field J. Nest inheritance is the missing source of direct fitness in a primitively eusocial insect. Science 2011;333:874-876.

60. Field J, Leadbeater E. Cooperation between non-relatives in a primitively eusocial paper wasp, Polistes dominula. Phil Trans Roy Soc B. 2016;371:20150093.

61. Bhadra A, Gadagkar R. We know that the wasps 'know': cryptic successors to the queen in Ropalidia marginata. Biol Lett. 2008;4:634-637.

62. Bang A, Gadagkar R. Reproductive queue without overt conflict in the primitively eusocial wasp Ropalidia marginata. Proc Nat Acad Sci USA. 2012;109:14494-14499.

63. Saito F, Kojima J-I: Colony cycle in the south-eastern coastal populations of Ropalidia plebeiana, the only Ropalidia wasp occurring in temperate Australia. Entomol Sci. 2005;8:263-275. 
64. Ohtsuki $\mathrm{H}$, Tsuji K. Adaptive reproduction schedule as a cause of worker policing in social Hymenoptera: A dynamic game analysis. Am Nat. 2009;173:747-758.

\section{Figures}

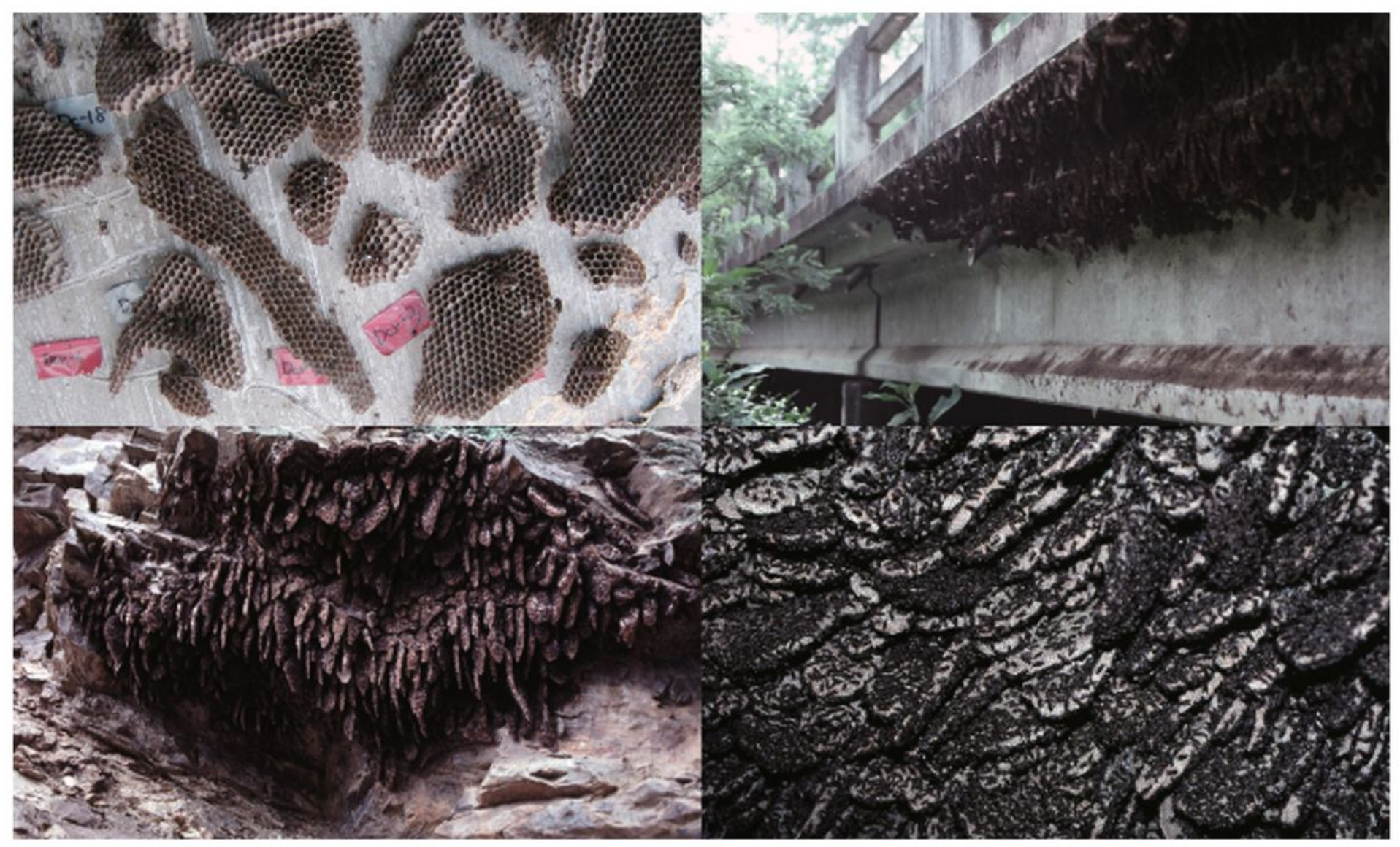

\section{Figure 1}

Nest aggregations of Ropalidia plebeiana. Upper left: Founding stage (October 2001) at Dinner Creek. Upper right: After worker emergence (February 2002) at Cabbage Tree Creek, the same place described by Richards [27]. Lower left: Nest aggregation (February 2002) at Cliff. Lower right: Nest aggregation at the reproductive-producing stage (March 2002) at Dinner Creek. Location names correspond to descriptions in Table S1. KT took all photographs. 

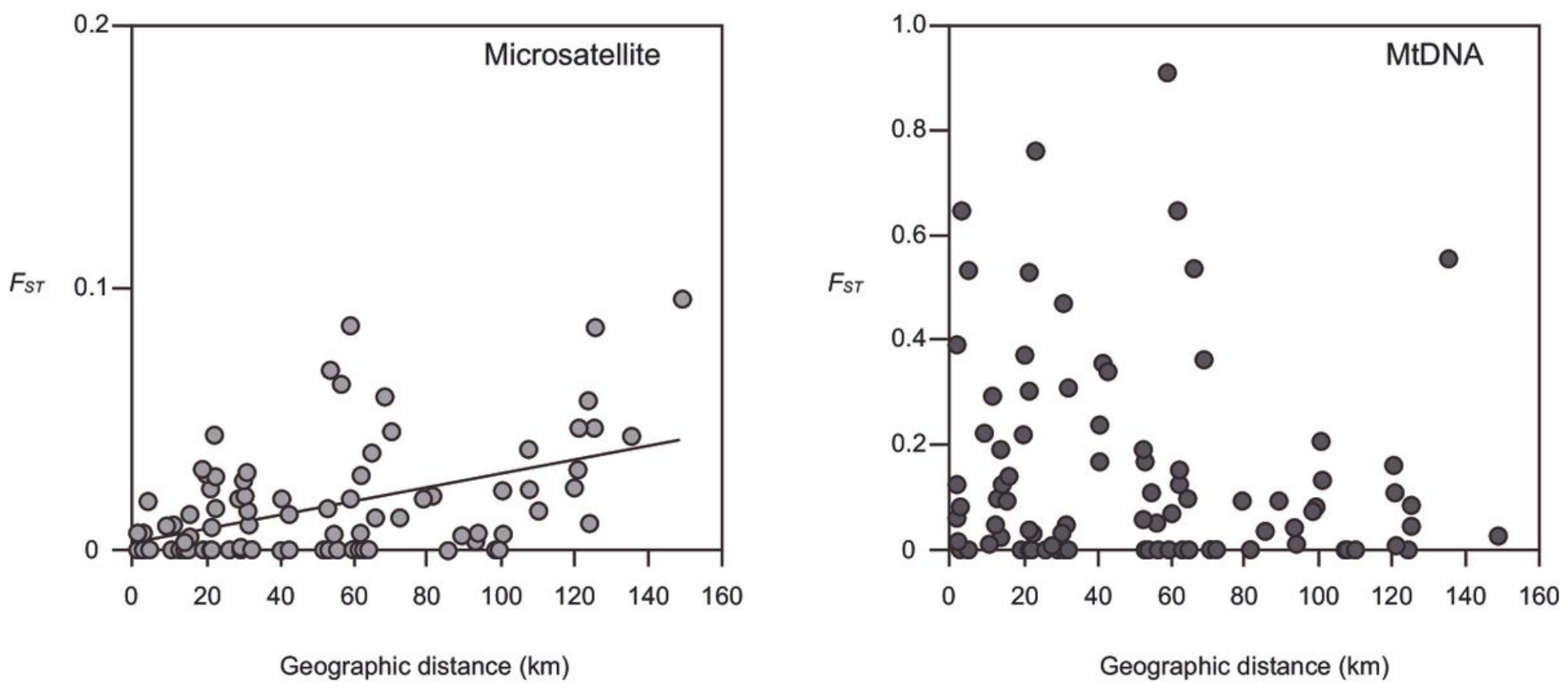

Figure 2

Isolation-by-distance relationships based on microsatellite and cytochrome oxidase subunit I (MtDNA) datasets. Using the Mantel test we found that the former relationship was significant ( $r s=0.497, P=$ $0.01)$ but the latter one was not $(r s=-0.014, P=0.51)$. 


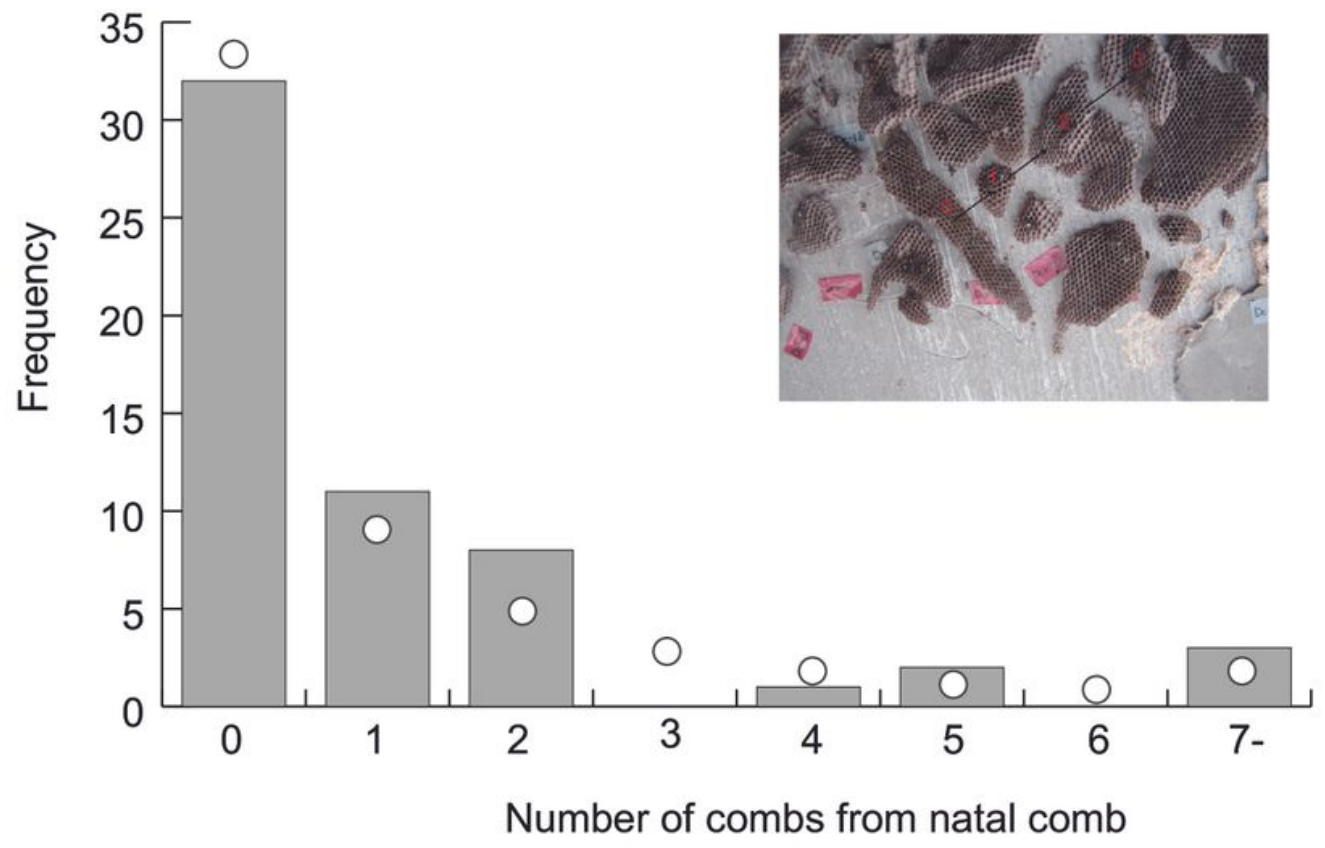

\section{Figure 3}

The number of individuals marked in the previous season that were found again in the next spring. The inset explains how to count the number of nests from the natal nest; 0 indicates a natal nest, and 3 indicates the third nest from the natal nest on which a focal foundress was found again in the next spring. We counted the least number of nests from the natal nest to the nest where the individual was found, and in this case, the number was 3 . The frequency distribution did not differ from a negative binomial distribution, representing a contagious distribution model. 


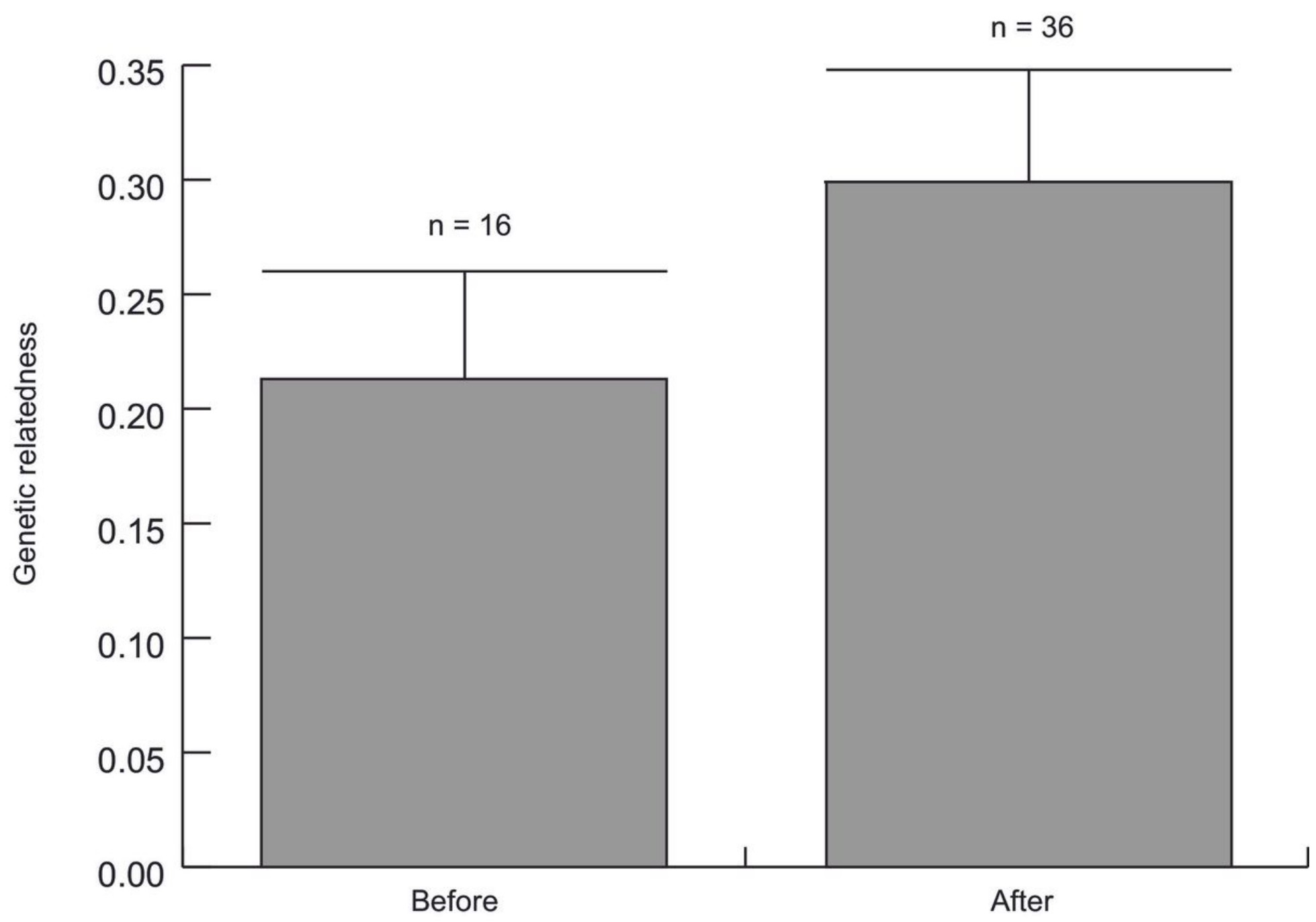

Figure 4

Genetic relatedness among foundresses before and after comb-cutting. Values for relatedness did not differ significantly (Welch's t-test, $\mathrm{P}=0.197$ ). 


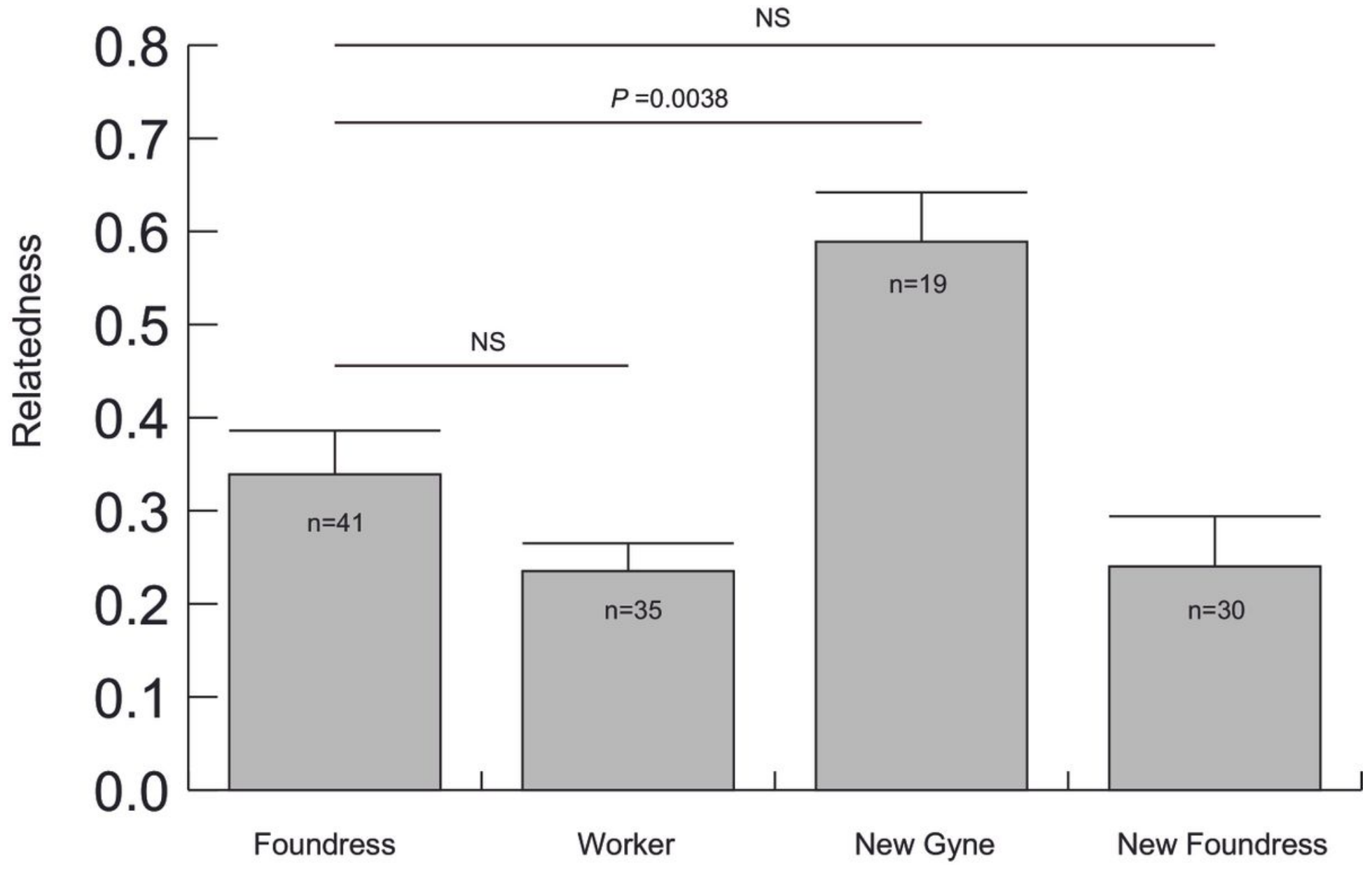

Figure 5

Genetic relatedness among each class of females: foundresses, workers, new gynes, and new foundresses in the next spring (i.e., individuals found the next spring). The value among foundresses differed significantly from that among new gynes using Welch's t-test, even after sequential Bonferroni correction. Sample sizes indicate the number of colonies used to calculate genetic relatedness. ns: nonsignificant with Welch's t-test after sequential Bonferroni correction. 


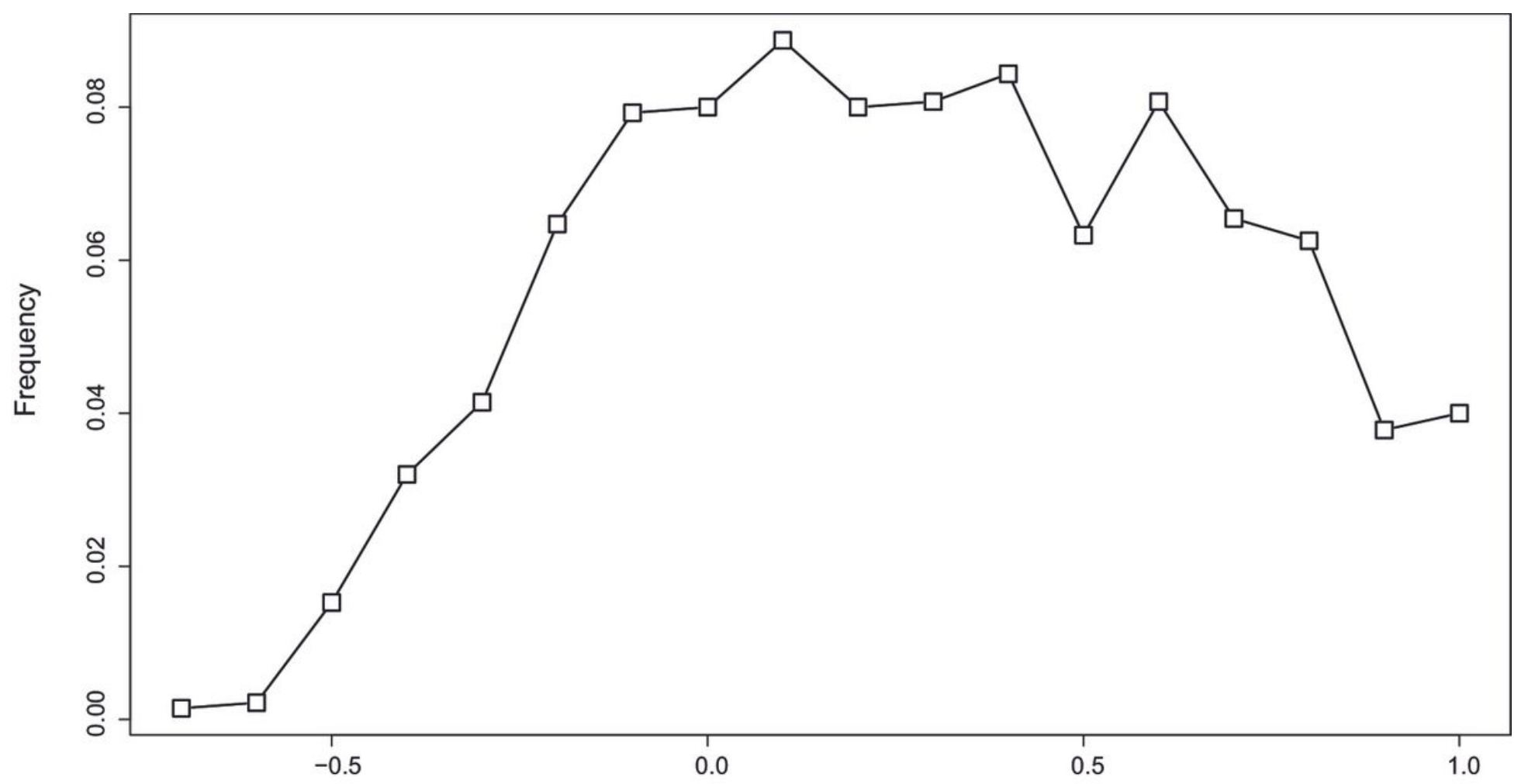

Pair-wise relatedness

Figure 6

Frequency distribution of pairwise relatedness among foundresses estimated using Kinship [36]. 


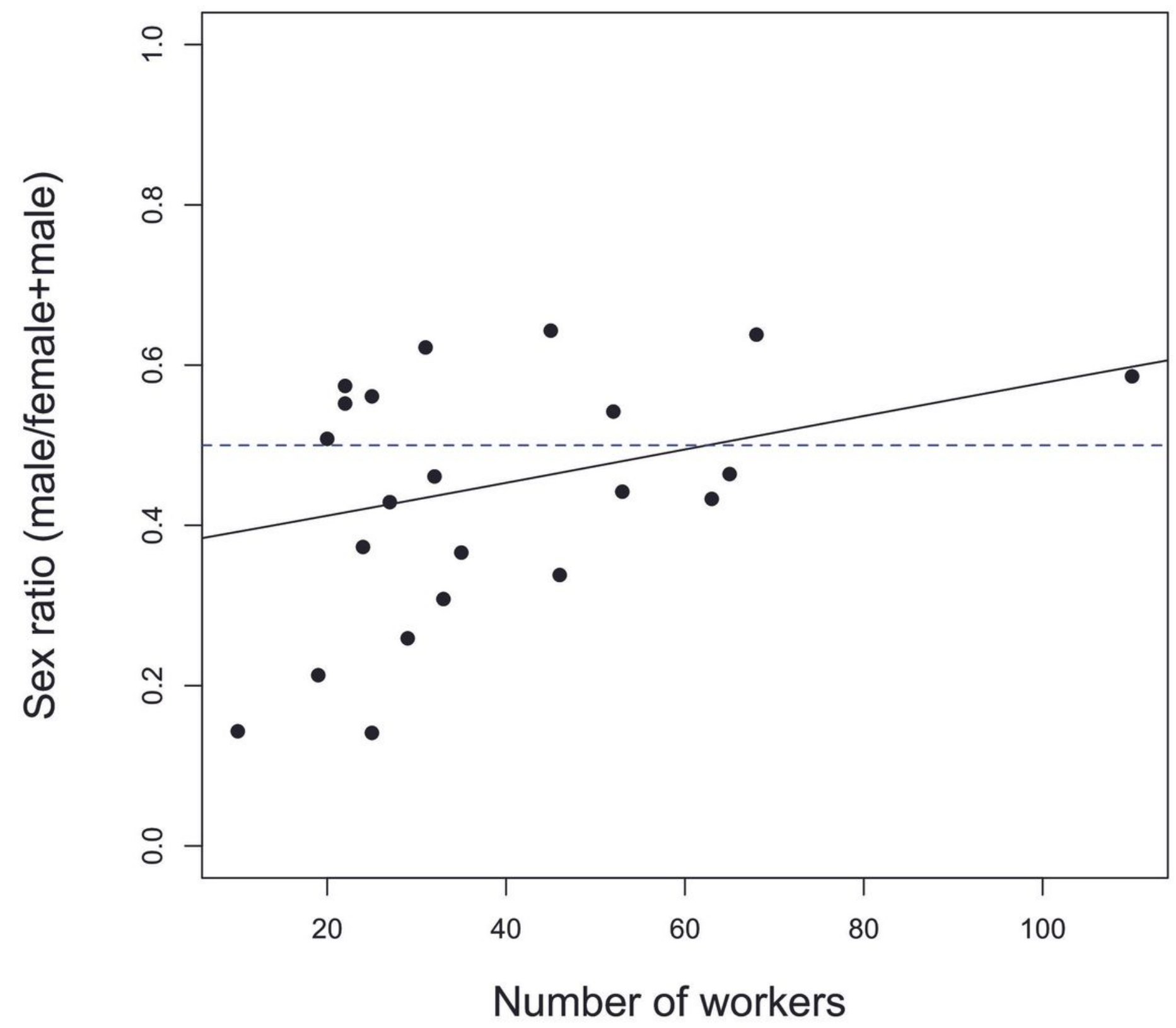

Figure 7

Relationship between the number of workers and sex ratio (proportion of males). The relationship was significant based on GLM analysis (see also Table S3). 


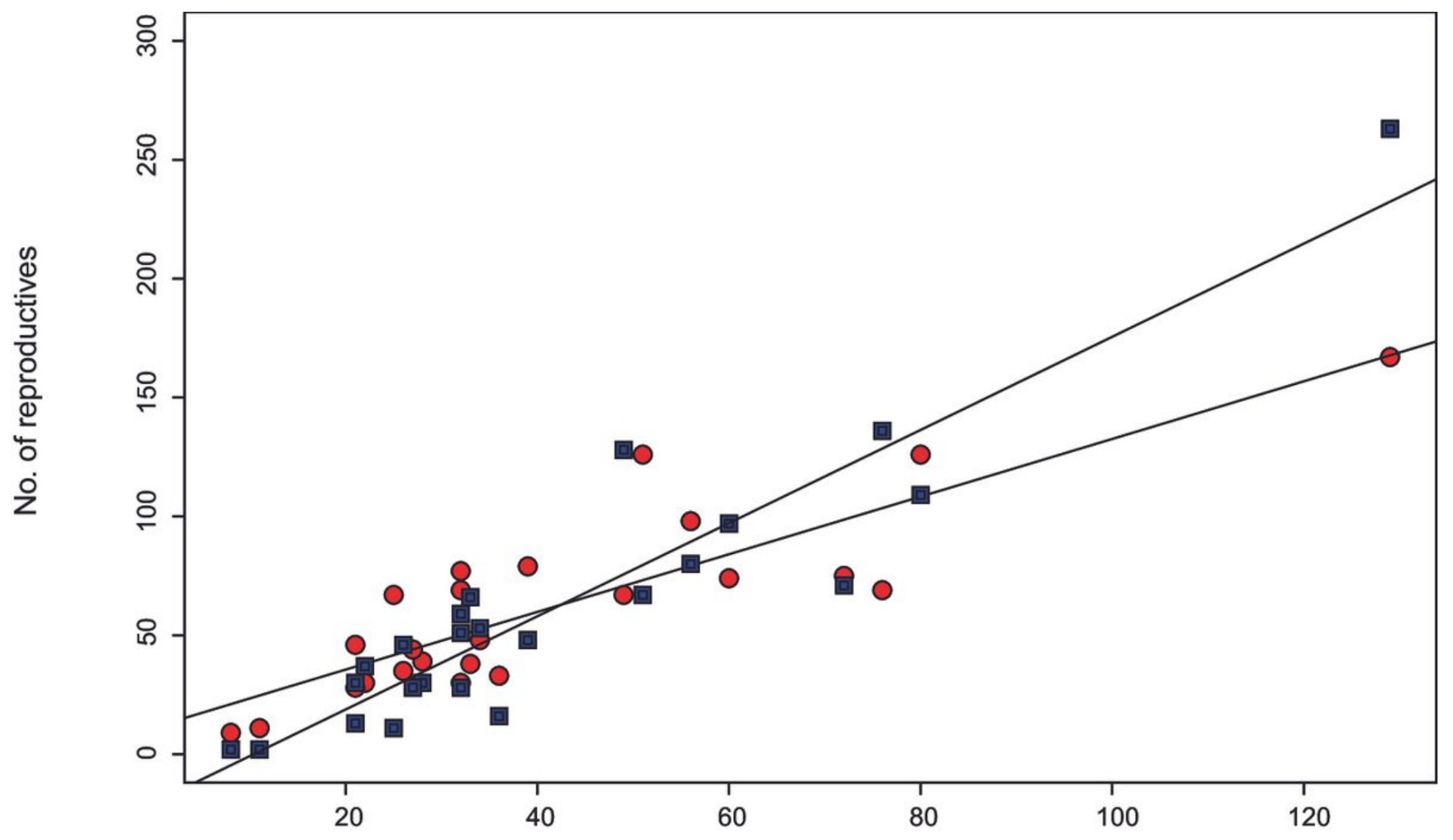

No. of workers

Figure 8

The relationships between the number of workers and new gynes (circles) and males (squares).

\section{Supplementary Files}

This is a list of supplementary files associated with this preprint. Click to download.

- FigS1Barplot.eps

- Tables1populations.docx

- Tables2primers.docx

- TableS3GLM.docx

- TableS4.docx 